\title{
Potential Tendency Differences between English and Japanese in Detecting Appropriate Respondents at Q\&A Sites
}

\author{
Yuya YOKOYAMA*, Teruhisa HOCHIN ** and Hiroki NOMIYA ** \\ * Kyoto Prefectural University, 1-5 Hangi-cho, Shimogamo, Sakyo-ku, Kyoto 606-8522, Japan \\ ** Kyoto Institute of Technology, Gosyokaidou-cho, Matsugasaki, Sakyo-ku, Kyoto, 606-8585, Japan
}

\begin{abstract}
In order to eliminate mismatches between the intentions of questioners and respondents of Question and Answer (Q\&A) sites, nine factors of impressions for Japanese and English statements have experimentally been obtained. Factor scores are then estimated by using the feature values of statements. So far the possibility of searching Japanese respondents capable of giving appropriate answers to a newly posted question has been established. It has been shown that the distance and the number of appearance may help us select users who can give appropriate answers to a question. In the similar fashion, this paper tries to find the possibility of detecting respondents who can appropriately answer a newly posted question at English Q\&A sites. As a result of analysis, while there were several users who iteratively gave answer statements for Japanese, there are only a small number of respondents who only posted at most two answers for English.
\end{abstract}

Keywords: Q\&A Sites, Factor Score, Euclidean Distance

\section{INTRODUCTION}

Recently, the number of people using Question and Answer (Q\&A) sites on the Internet has been increasing. Q\&A sites are online communities where users can manually post questions and answers. Thus, these sites are thought to be databases containing enormous amounts of knowledge to solve various problems. When a user posts a question, others may respond. The questioner selects the most appropriate response as the "Best Answer" (BA) and awards the respondent with a certain number of points that serve as a fee. The BA is the response statement the questioner subjectively finds most satisfying.

As the number of users of Q\&A sites increases, and more questions are posted, it becomes harder for respondents to select questions that match their specialty and interests. Consequently, a question posed by a user may not be seen or answered by qualified respondents. Moreover, if an appropriate respondent is not encountered, mismatching may occur, causing the following problems: - A questioner may acquire incorrect knowledge from inappropriate answers.

- Respondents may not have the necessary knowledge to properly answer the question, and thus the problem remains unsolved.

- Users may be offended by answers that contain abusive words, slanders, or statements against public order and standards of decency.

There have been a number of prior works investigating Q\&A sites; introducing users to answer statements $[1,2]$, and inspecting the quality or tendency of answer statements [3-5], etc. These prior works have mainly used textual features or link analysis. However, some users may prefer a polite style, while others may write statements in a ruder style. Some commonly use abstract words, whereas others use more concrete words. These tendencies have not been considered. Meanwhile, our work focuses on using impressions as well as textual features. Moreover, although there are some prior works that introduce users to answer statements as described [1, 2], it is difficult to say that a way to introduce appropriate respondents to a questioner has yet been established. Therefore, with using the impression of statements, our work aims to introduce appropriate respondents to a questioner.

Our goal is to present questions to users who are qualified to properly answer them, thus avoiding the problems described above. The impressions of sixty statements posted on Yahoo! Chiebukuro (Y!C) [6] have been evaluated [7]. By applying factor analysis to the experimental results, nine factors were obtained.

Factor scores obtained through factor analysis represent the impressions of the statements, and this is necessary for estimating the factor scores of other statements. The statements were estimated using multiple regression on the feature values assigned to the statements [8]. The overall estimation accuracy for all nine factors was proved to be good.

The possibility of detecting respondents who can appropriately answer a newly posted Japanese question was examined [9]. Here we assumed that a respondent whose impression is similar to that of a questioner is 
thought to be adequate to give an adequate answer. We attempted a process of getting proper respondents to a question by using questions actually already posted at Y!C. As a result of analysis, it was shown that there were some nonresponding users who have a shorter Euclidean distance between the factor score of a questioner and that of a respondent. Those users were thought to be capable of appropriately answering that question. It was also shown that with the consideration of categories, more appropriate users could be detected [10].

Most of the feature values, however, are dependent on Japanese. Therefore, it is necessary to look into how extensive our method can be applied in other languages as well [11]. Experimental materials evaluated were the statements actually posted to Yahoo! Answers (Y!A) [12]. As a result, nine factors were obtained. It has been shown that some main factors could be obtained in English as well. It has also been shown that different type of factors could be obtained from English statements. Factor scores were then estimated from the feature values of English statements, such as words and characters, syntactic information, and appearance percentages through multiple regression analysis [13]. As a result of estimation, it has been shown that estimation accuracies of all of the factors are very good.

In this paper, in the similar fashion as Japanese, we try to find the possibility of detecting respondents who can appropriately answer a newly posted English question at Y!A. As a result of analysis, some different tendency could be observed between English and Japanese; among the 100 short distances between question and answer statements, while there are several users who iteratively posted answer statements for Y!C (Japanese), there are only a few respondents who give at most two answers for Y!A (English). This could result from customary or cultural difference between English and Japanese.

The remainder of the paper is organized as follows. Our previous works are summarized in Section 2. A method to find appropriate potential answerers is proposed and evaluated in Section 3. Considerations towards our analysis are provided in Section 4. Finally, Section 5 concludes the paper.

\section{PREVIOUS WORKS}

\subsection{Obtaining Factors of Statements (Japanese)}

An experiment was conducted to evaluate impressions of answers. There were forty-one evaluators, and they evaluated the style or content of statements and assigned labels from a group of fifty words [7]. Twelve sets of questions and answers were evaluated, and these included three from each of four major categories: Auction, PC, Love, and Political/Social Problems; the categories were chosen from those actually posted at $\mathrm{Y}$ !C in 2005 [7]. Factor analysis was applied to the experimental results, and nine factors were obtained. The factors indicate the nature of a statements, as explained by the various impression words assigned to that statement; they were named accuracy, displeasure, creativity, ease, persistence, ambiguity, moving, effort, and hotness.

\subsection{Estimation of Factor Scores (Japanese)}

\subsubsection{Feature Values of Statements}

The factor scores were obtained for only the sixty statements used in the experiment. To be able to estimate the factor scores of other statements, multiple regression analysis was applied to their feature values [8]. Overall, seventy-seven feature values were adopted; these are summarized, syntactic information, word imageability, expression in the closing sentence, word familiarity and notation validity.

\subsubsection{Estimation Result}

Multiple regression analysis was performed on the sixty questions and answers employed in the impression evaluation experiment. 281 quadratic terms (the product of two explanatory variables) were used according to seventy-seven explanatory variables, and the respondent variables with factor scores for the nine factors.

Multiple correlation coefficients, which show the goodness of the estimation, were above 0.9 for all nine factors [8]. As a result of analysis, it was shown that the estimation accuracies of all of the factors are very good.

\subsection{Impression and Suitability of Q\&A (Japanese)}

\subsubsection{Purpose}

The differences are determined between the impressions of a given question and the answers already posted for it in $\mathrm{Y}$ !C, by calculating the Euclidean distance between their factor scores [9]. These differences could be used to identify those users who could appropriately answer that question.

\subsubsection{Dataset}

The differences between the impressions of the questions and those of the answers, and the suitability of the answers were examined in order to determine if it would be possible to use this information to find users who would be expected to give an appropriate answer [9]. One question was chosen from each of the following categories: Auction, PC, and Love. For the respondents 
who posted the answers to those questions, the factor scores for most of the answers they posted in 2005 are obtained, by using the multiple regression equations described in Section 2.2.2.

\subsubsection{Result}

Distances were calculated for a total of 66,238 answer statements, using the formula (1):

$$
D=\sqrt{\sum_{k=1}^{9}\left(F a c_{Q_{k}}-F a c_{A_{k}}\right)^{2}}
$$

where $F a c_{Q_{k}} F a c_{A_{k}}$ are the score for the kth factor for a question and for an answer, respectively. The distances were then sorted in ascending order. The threshold rank is determined based on the irregular bump. Each threshold rank is set as 24, 18, and 22 for the Auction, PC, and Love statements, respectively.

The answer statements whose ranks are smaller or equal to each threshold one are shown in Table 1. Each row indicates a separate answer statement other than one actually posted to its question used for the analysis. Each column entitled is explained as follows:

- "No." means the ascending order rank of distance for each answer statement.

- "Answer" indicates whether the respondents gave an answer to the questions used for the analysis. Each answer statement is given either notation such as "A01" or "Others." How to interpret these notations are explained as follows:

○ "A," "P," and "L" are denoted if they answered the questions, standing for Auction, PC, and Love, respectively, regardless of the identical category.

○ Numbers are given from "01" to "09" ("06," respectively) for Auction (PC and Love).

- The number " 01 " means the answer statement actually chosen as a BA by the questioner.

- The numbers "03," “04," “05," “06," “07," and " 09 " indicate the answer statements of non-BAs denoted as actually posted in chronological order. The numbers " 02 " and " 08 " happen to be absent in Table 1.

- "Others" is denoted if they did not actually answer the questions.

- "Distance" means the Euclidean distance between the factor score of the answer statement and that of a question one, obtained through Formula (1).

- "ID" means Q\&A page IDs where each answer statement is posted and browsed. IDs consist of unique multiple digit numbers. Each page can be referred in the URL composed of "http://detail.chiebukuro.yahoo.co.j p/qa/question_detail/q" and a respective ID.

- "Overlap" indicates users who appear at least twice. Those users are denoted as (A) to (E). They are shaded in Table 1 to emphasize that they appear at least twice.

How to grasp the data in Table 1 will be explained by taking an example of answer statements denoted as "A01." The statements are posted by the same respondent (A) whose one actual answer to an Auction question was actually chosen as a BA. Among those answer statements other than the BA, the second (third, respectively) row of Auction indicates that the distance is 0.796 (0.805) between the Auction question and an answer whose ID is 111505126 (12961032) posted by (A).

An answer statement actually posted by a user who also answered a question of one category can appear in another dataset of a different category. Taking an example of (A), an answer statement denoted as "A01," whose distance is 0.999, appears in the PC dataset, though it was posted by (A), who answered an Auction question.

\subsubsection{Analysis}

The users denoted as (A), (C), and (E) in the column entitled "Overlap" appear many times for Auction, PC, and Love, respectively. Thus users who gave answer statements of the same categories appear in Table 1. From these results, with the consideration of categories of answer statements, particularly some users appear most in each dataset [10]. Therefore, users who have posted answers on a certain category can be thought to appropriately answer a question of the identical category. It can be regarded that a specific user who specializes in a particular category could be expected to give appropriate answers to questions of the identical category. It can be regarded that a specific user who specializes in a particular category could be expected to give appropriate answers to questions of the identical category.

\subsection{Obtaining Factors of Statements (English)}

Most of the feature values in our study are dependent on Japanese. Thus, it is required to see how extensive our study could be applied in other languages. As a first step, factors that describe impression of English statements were obtained in the similar fashion stated in Section 2.1.

In order to directly compare our English method with Japanese one, most of the impression words used are the same ones [11]. With using these fifty impression words, 
Table 1 Answer statements whose factor scores are close to those of the question (Japanese)

\begin{tabular}{|c|c|c|c|c|}
\hline No. & Answer & Distance & ID & Overlap \\
\hline 1 & A03 & 0.770 & 13228478 & \\
\hline 2 & A01 & 0.796 & 111505126 & (A) \\
\hline 3 & A01 & 0.805 & 12961032 & (A) \\
\hline 4 & A04 & 0.831 & 12402382 & (B) \\
\hline 5 & A01 & 0.834 & 12847837 & (A) \\
\hline 6 & A01 & 0.862 & 12617472 & (A) \\
\hline 7 & A09 & 0.899 & 114526801 & \\
\hline 8 & Others & 0.910 & 1038895 & \\
\hline 9 & A01 & 0.913 & 126678687 & (A) \\
\hline 10 & A01 & 0.914 & 132071733 & (A) \\
\hline 11 & A01 & 0.933 & 143239349 & (A) \\
\hline 12 & A01 & 0.935 & 135925478 & (A) \\
\hline 13 & Others & 0.939 & 1031505 & \\
\hline 14 & A04 & 0.943 & 11181606 & (B) \\
\hline 15 & A01 & 0.944 & 132089723 & (A) \\
\hline 16 & A01 & 0.946 & 13624073 & (A) \\
\hline 17 & Others & 0.948 & 1036190 & \\
\hline 18 & A01 & 0.952 & 102620885 & (A) \\
\hline 19 & $\mathrm{~A} 01$ & 0.955 & 121809722 & (A) \\
\hline 20 & A01 & 0.960 & 146442314 & (A) \\
\hline 21 & A04 & 0.966 & 10294125 & (B) \\
\hline 22 & A07 & 0.972 & 12350617 & \\
\hline 23 & A01 & 0.975 & 132702838 & (A) \\
\hline 24 & A01 & 0.976 & 132678538 & (A) \\
\hline
\end{tabular}

(b) $\mathrm{PC}$

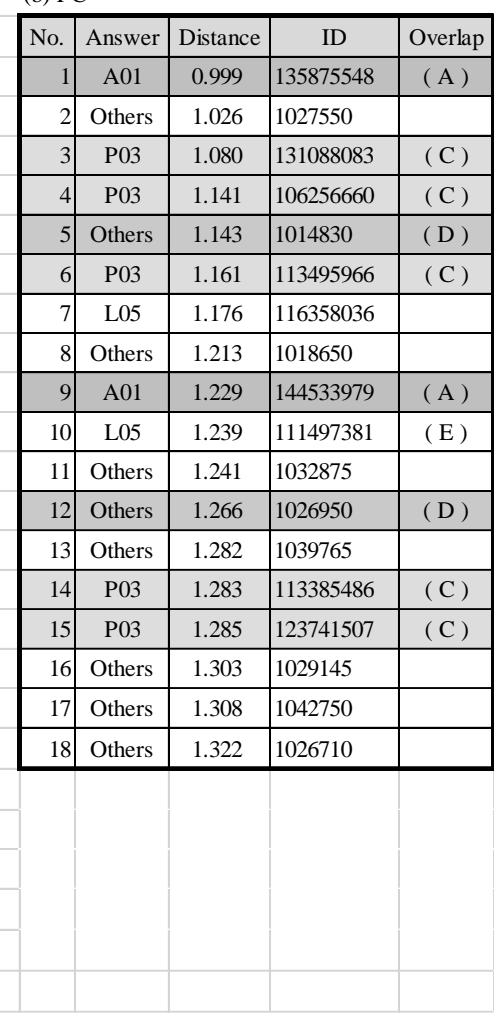

(c) Love

\begin{tabular}{|r|c|c|l|c|}
\hline No. & Answer & Distance & \multicolumn{1}{|c|}{ ID } & Overlap \\
\hline 1 & L05 & 0.850 & 10937970 & ( E ) \\
\hline 2 & L06 & 0.864 & 131363453 & \\
\hline 3 & L05 & 0.875 & 11652216 & ( E ) \\
\hline 4 & L05 & 0.925 & 11795306 & ( E ) \\
\hline 5 & L05 & 0.930 & 136283433 & ( E ) \\
\hline 6 & L05 & 0.934 & 122611942 & ( E ) \\
\hline 7 & L05 & 0.939 & 13807818 & (E) \\
\hline 8 & L05 & 0.941 & 101124475 & (E) \\
\hline 9 & L05 & 0.944 & 111178621 & (E) \\
\hline 10 & Others & 0.945 & 1029535 & \\
\hline 11 & L05 & 0.949 & 112562681 & (E) \\
\hline 12 & L05 & 0.953 & 102295340 & (E) \\
\hline 13 & Others & 0.961 & 1046935 & \\
\hline 14 & Others & 0.962 & 1046515 & \\
\hline 15 & L05 & 0.966 & 122938652 & (E) \\
\hline 16 & L06 & 0.990 & 131191763 & \\
\hline 17 & L05 & 1.004 & 101178825 & (E) \\
\hline 18 & L05 & 1.008 & 12382852 & (E) \\
\hline 19 & L04 & 1.015 & 133649808 & \\
\hline 20 & L05 & 1.020 & 131041513 & (E) \\
\hline 21 & A01 & 1.030 & 125454957 & ( A ) \\
\hline 22 & L05 & 1.030 & 12522997 & (E) \\
\hline
\end{tabular}

impression evaluation experiment was conducted for four foreign subjects (3 males: Mexican, Vietnamese, and French, and 1 female: French). Experiment materials were six sets of Q\&A English statements from Y!A (three each from "Computer \& Internet" and "Auction ebay."). Similar as the previous experiment depicted in Section 2.1, each set consists of one question and four answer statements including the "BA."

Factor analysis was applied to the experimental results. As a result of the analysis, nine factors were obtained. Both similarity and difference were confirmed between the Japanese factors and English ones. Moreover, some factors could be obtained in one language while they failed to in another language.

\subsection{Estimation of Factor Scores (English)}

Similar as the estimation of Japanese factor scores explained in Section 2.2, multiple regression analysis was applied to the feature values in order to be able to estimate factor scores of all the statements [13]. Overall, twenty-six feature values were adopted. These are summarized, as follows:

- Word: The appearance number of word of each statement is extracted. Moreover, the ratio of word to sentence is taken into consideration as well.
- Part-of-Speech (POS): Syntactic information was extracted through morphological analysis applied to the statements of experimental materials used in Section 2.4. Similar as Word depicted above, the appearance number and ratio of POS of each statement is extracted.

As a result of analysis, the estimation of all the nine factors were very good.

\section{IMPRESSION and SUITABILITY of Q\&A (English)}

\subsection{Purpose}

As explained in Section 2.3, the differences are determined between the impressions of a given question and the answers already posted for it in $\mathrm{Y}$ !C, by calculating the Euclidean distance between their Japanese factor scores [9, 10]. These differences could be used to identify those users who could appropriately answer that question. In order to see how this method could be extensive in English, the differences determined by impressions will be applied to English statements as well.

\subsection{Dataset}

The differences between the impressions of the English questions and those of the answers, and the suitability of the answers are examined in order to determine if it would be possible to use this information to 
Table 2 Answer statements whose factor scores are close to those of the question (English)

\begin{tabular}{|c|c|c|c|c|c|c|c|}
\hline \multicolumn{4}{|c|}{ (a) QEng01 } & \multicolumn{4}{|c|}{ (b) QEng02 } \\
\hline No. & Distance & ID & Overlap & No. & Distance & ID & Overlap \\
\hline 1 & 1.38 & 20100301190221AAHGC5Q & & 1 & 1.54 & 20100328095230AAhycCR & \\
\hline$\ldots$ & $\ldots$ & $\ldots$ & $\ldots$ & $\ldots$ & $\ldots$ & $\ldots$ & $\ldots$ \\
\hline 47 & 2.79 & 20100221140530AAlcqiL & (A) & 27 & 2.50 & 20100328094946AAMZV xy & (D) \\
\hline 48 & 2.79 & 20100221140530AAlcqiL & (A) & $\ldots$ & $\ldots$ & $\ldots$ & $\ldots$ \\
\hline$\ldots$ & $\ldots$ & $\ldots$ & $\ldots$ & 69 & 3.09 & 20100227123651AA21OkR & (E) \\
\hline 79 & 3.09 & 20100223144245AAtS42Q & (B) & $\ldots$ & $\ldots$ & $\ldots$ & $\ldots$ \\
\hline 80 & 3.09 & 20100223144245AAtS42Q & (B) & 88 & 3.26 & 20100327093527AALC1du & (D) \\
\hline 81 & 3.11 & 20100225191231AAliD6y & (C) & & $\ldots$ & & $\ldots$ \\
\hline$\ldots$ & $\ldots$ & $\ldots$ & $\ldots$ & 92 & 3.27 & 20100227123651AA21OkR & (E) \\
\hline 86 & 3.13 & 20100225191231AAIID6y & (C) & $\ldots$ & $\ldots$ & $\ldots$ & $\ldots$ \\
\hline$\ldots$ & $\ldots$ & $\ldots$ & $\ldots$ & & & & \\
\hline & & & & & & & \\
\hline
\end{tabular}

find users who would be expected to give an appropriate answer $[9,10]$. Experimental materials are extracted from sourceforge [14], where data on Y!A are open to public so that research on Community Question Answering can be facilitated. Since the category of all the Q\&A statements provided in sourceforge [14] is only "Computer \& Internet," the category of three questions and answer statements used for the analysis is "Computer \& Internet."

For the respondents who posted the answers to those questions, the factor scores for most of the answers they posted in 2013 are obtained, by using the multiple regression equations, as described in Section 2.5. Factor scores are calculated using Java programs. The factor scores calculated are three question and altogether 5,491 answer statements. Those statements extracted are as follows:

- Three question statements used for the analysis are randomly chosen. However, the statements are chosen that include as few grammatical errors and miswritten characters as possible so that morphological analysis can be applied without problems.

- The following total 5,491 answer statements are extracted from sourceforge [14].

- Answer statements are chosen that are posted by the respondents who gave answers to each question statement used for the previous experiment depicted in Section 2.3.4. The total number of these answer statements is 313 .

- Answer statements are selected that are posted by the respondents who did not post answers to any question statements used in Section 2.3.4. The number of these answer statements is 5,178 .

\subsection{Results}

Euclidean distances $D$ between each question statement and 5,491 answer statements are calculated by using the formula (1), as explained in Section 2.3.3. The distances are then sorted in ascending order.
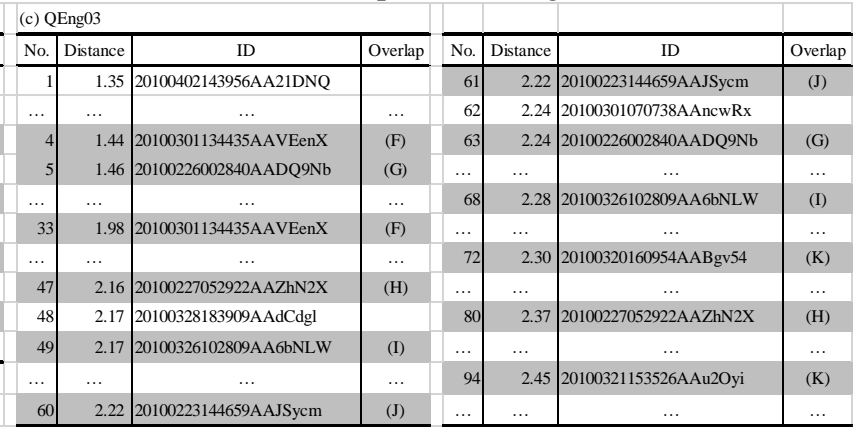

The answer statements whose distances are ranked in shorter 100 are shown in Table 2. A column "ID" that does not appear in previous result (Table 1) are explained as follows. Columns entitled "No.," "Distance" and "Overlap" are depicted in Section 2.3.3.

- "ID": IDs are composed of the mixture of alphabets numbers, starting from eight numbers consisting of the date of actual posted time. Each page can be referred and browsed in the URL comprising "http:// answers. yahoo.com/question/index?qid=" and a respective ID.

\subsection{Analysis}

From the results shown in Table 2, the numbers of users who actually answered each question are 3, 2, and 6, respectively. Unlike the previous result shown in Table 1, where particular users appear repeatedly in short distance, only a few users appear at most twice. Thus, differences of tendency can be observed between Japanese and English in finding appropriate respondents at Q\&A site.

\section{CONSIDERATIONS}

Several certain respondents frequently appeared for Y!C, as shown in Table 1 (Japanese), while mere a handful of respondents appeared not more than twice for Y!A, as shown in Table 2 (English). There are two possible reasons. Firstly, the category used for those analyses and included in respective dataset are completely different. For Y!C dataset, provided by National Informatics of Institute [15], it covers most of the categories in Y!C. On the other hand, for Y!A dataset, provided as sourceforge [14], it includes only the category "Computer \& Internet." Thus, it is required to add for other categories than "Computer \& Internet" for Y!A.

Another possible reason is that there could be customary or cultural difference between English and Japanese. In calculating factor scores, the meanings of contents are not regarded. Therefore, in order to inspect the differences more profoundly, the contents of answer 
statements should be minutely regarded and examined. Further analysis could shed the light on customary or cultural difference between English and Japanese.

\section{CONCLUSION}

In this paper, in the similar fashion as Japanese, we tried to find the possibility of finding respondents who can appropriately answer a newly posted English question at Y!A. As a result, some different tendency could be observed between English and Japanese. While there were several users who iteratively gave answer statements for $\mathrm{Y} ! \mathrm{C}$ (Japanese), there are only a small number of respondents who only posted at most two answers for $\mathrm{Y}$ !A (English). This could result from customary or cultural difference between English and Japanese.

For future work, an additional experiment with more subjects and samples is required. Since all the foreign subjects were non-native English, an experiment with native English subjects must be conducted. The contents must be regarded so that customary or cultural difference between English and Japanese could be revealed. It is also required to use the estimated scores to estimate the BAs. To detect appropriate answerers, the characteristics of users must be revealed and used for investigation.

\section{ACKNOWLEDGMENTS}

This research was partially supported by the Japan Society for the Promotion of Science, Grant Number 26008587, and 2015-2016.

\section{REFFERENCES}

1. Pawal Jurczyk and Eugene Agichtein: Discovering Authorities in Question Answer Communities by Using Link Analysis, Proc. of 16th ACM Conference on Information and Knowledge Management (CIKM), pp. 919-922, 2007.

2. Fatemeh Riahi, Zainab Zolaktaf, Mahdi Shafiei and Evangelos Milios: Finding Expert users in Community Question Answering, Proc. of the 21st International Conference Companion on World Wide Web (WWW12), pp.791-798, 2012.

3. Eugene Agichtein, Castillo Carlos, Debora Donato, Aristides Gionis and Mishne Gilad: Finding High-Quality Content in Social Media, Proc. of the Int'l Conf. on Web Search and Web Data Mining (WSDM08), pp.183-194, 2008.

4. Yuanjie Liu, Shasha Li, Yunbo Cao, Chin-yew Lin, Dingyi Han, and Yong Yu: Understanding and Summarizing Answers in Community-Based Question Answering Services, Proc. of the 22nd
International Conference on Computational Linguistics, pp.497-504, 2008.

5. Daphne Ruth Raban: Self-Presentation and the Value of Information in Q\&A Websites, Journal of the American Society for Information Science and Technology Volume 60, Issue 12, pp.2465-2473, 2009.

6. Yahoo! Chiebukuro (URL, in Japanese), http://chiebukuro.yahoo.co.jp/, 2018.

7. Yuya Yokoyama, Teruhisa Hochin, Hiroki Nomiya, and Tetsuji Satoh: Obtaining Factors Describing Impression of Questions and Answers and Estimation of their Scores from Feature Values of Statements, Software and Network Engineering, pp.1-13, Springer, 2012.

8. Yuya Yokoyama, Teruhisa Hochin, and Hiroki Nomiya: Using Feature Values of Statements to Improve the Estimation Accuracy of Factor Scores of Impressions of Question and Answer Statements, International Journal of Affective Engineering, Vol. 13, No. 1, pp.19-26, 2014.

9. Yuya Yokoyama, Teruhisa Hochin, and Hiroki Nomiya: Towards Detecting Appropriate Respondents to Questions Posted at Q\&A Sites by Disregarding and Considering Categories of Answer Statements, International Journal of Affective Engineering, Vol.15, No.2, pp.167-175, 2016.

10. Yuya Yokoyama, Teruhisa Hochin, and Hiroki Nomiya: Improvement of Obtaining Potential Appropriate Respondents to Questions at Q\&A Sites by Considering Categories of Answer Statements, International Journal of Affective Engineering, Vol.16, No.2, pp.63-73, 2017.

11. Yuya Yokoyama, Teruhisa Hochin, and Hiroki Nomiya: Factors Describing Impression of English Question and Answer Statements, Proc. of 11th Spring Conference of Japan Society of Kansei Engineering, G12-3, 2016.

12. Yahoo! Answers (URL), https://answers.yahoo.com, 2018.

13. Yuya Yokoyama, Teruhisa Hochin, and Hiroki Nomiya: Estimation of Factor Scores from Feature Values of English Question and Answer Statements, Proc. of 15th IEEE/ACIS International Conference on Computer and Information Science (ICIS2016), pp.741-746, 2016.

14. sourceforge, Yahoo! Answers Datasets, Summary (URL), http://sourceforge.net/projects/yahoodataset/, 2018.

15. National Institute of Informatics (URL, in Japanese), http://www.nii.ac.jp, 2018. 